This item was submitted to Loughborough's Institutional Repository (https://dspace.lboro.ac.uk/) by the author and is made available under the following Creative Commons Licence conditions.

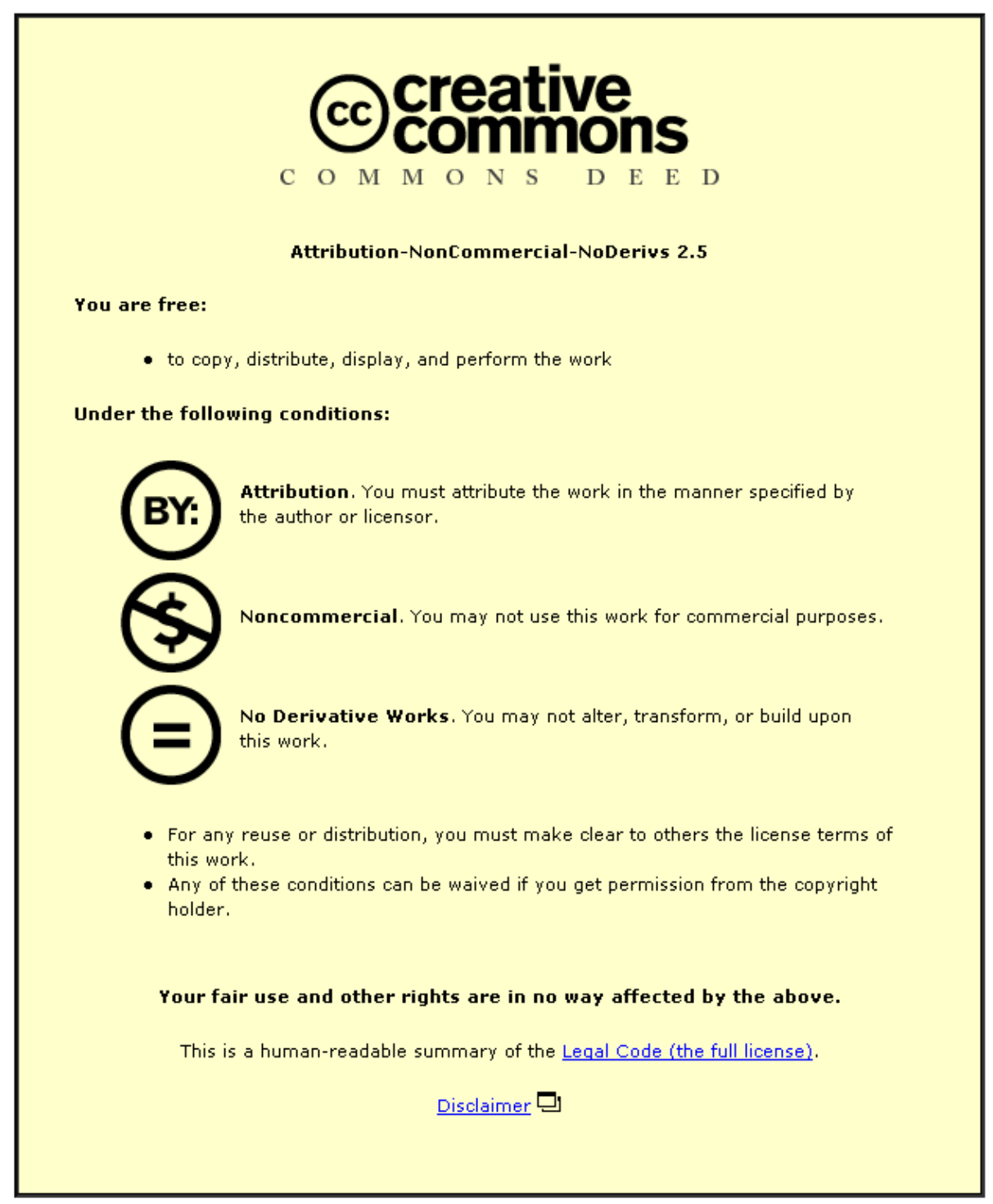

For the full text of this licence, please go to: http://creativecommons.org/licenses/by-nc-nd/2.5/ 


\title{
The Effect of Cost Function on Optimum Technique of the Undersomersault on Parallel Bars
}

\author{
Michael J. Hiley and Maurice R. Yeadon \\ Loughborough University
}

\begin{abstract}
The undersomersault, or felge, to handstand on parallel bars has become an important skill in Men's Artistic Gymnastics as it forms the basis of many complex variations. To receive no deductions from the judges, the undersomersault must be performed without demonstrating the use of strength to achieve the final handstand position. Two male gymnasts each performed nine undersomersaults from handstand to handstand while data were recorded using an automatic motion capture system. The highest and lowest scoring trials of each gymnast, as determined by four international judges, were chosen for further analysis. Three optimization criteria were used to generate undersomersault technique during the swing phase of the skill using a computer simulation model: minimization of peak joint torques, minimization of horizontal velocity before release, and maximization of angular momentum. The techniques used by both gymnasts could be explained using the second optimization criterion which facilitated further skill development. The first optimization criterion generated a technique advocated for beginners where strength might be expected to be a limiting factor. The third optimization criterion resulted in a different type of undersomersault movement of greater difficulty according to the FIG Code of Points.
\end{abstract}

Keywords: gymnastics, optimization, simulation

The undersomersault, or felge, from handstand to handstand (Figure 1) on parallel bars is an important skill in Men's Artistic Gymnastics as it provides the basis for more complex variations. From handstand the gymnast lowers the body by closing the shoulder angle (shoulder extension) and allowing the shoulders to move forward relative to the hands (Figure 1A-B). The gymnast then rotates backward and circles below the bar (Figure 1B-D). Release occurs after the gymnast's mass center has passed above bar level (Figure 1E-F) and the gymnast then regrasps the bars before reaching the handstand position (Figure 1G). To receive no deductions from the judges, the gymnast must perform the undersomersault without demonstrating the use of strength to achieve the final position. The gymnast should regrasp without flexing the elbows and should arrive in handstand without excessive flexion of the shoulders once the bars have been caught.

Two techniques for the swing phase (Figure 1B-F) of the undersomersault have been identified in the coaching literature (Davis, 2005). The technique depicted (Figure 1) in the Code of Points (FIG, 2006) closely resembles a backward clear circle to handstand, which is normally

Michael J. Hiley (Corresponding Author) and Maurice R. Yeadon are with the School of Sport, Exercise and Health Sciences, Loughborough University, Loughborough, U.K. performed on the high bar. During this technique the gymnast maintains the hip angle throughout the majority of the circle, in particular while the gymnast is below the bars (Figure 1). Davis (2005) recommended that this technique be used during the initial stages of learning the undersomersault. Presumably this technique is recommended for young gymnasts as it is less demanding in terms of the strength required to perform the skill.

However, the technique preferred by the majority of senior gymnasts more closely resembles a "stoop stalder" on high bar (Davis, 2005). As the gymnast circles beneath the bars a deep pike position (large hip flexion) is adopted from which the gymnast rapidly extends passing through release (Figure 2). It is speculated that the stoop stalder technique produces a more vertical path of the mass center near release, which would have advantages for both consistency and skill progression. The advantages of such a mass center path are as follows: firstly the direction of the mass center velocity changes less near to release leading to a more consistent performance. That is, if the release is mistimed slightly the mass center will still be moving in the correct direction (Hiley \& Yeadon, 2003). Secondly, the undersomersault to handstand forms the basis of more complex skills: the undersomersault to handstand with a half or full twist. Having a vertical mass center velocity while the body is twisting will reduce the complexity of the hand grasp changes. 


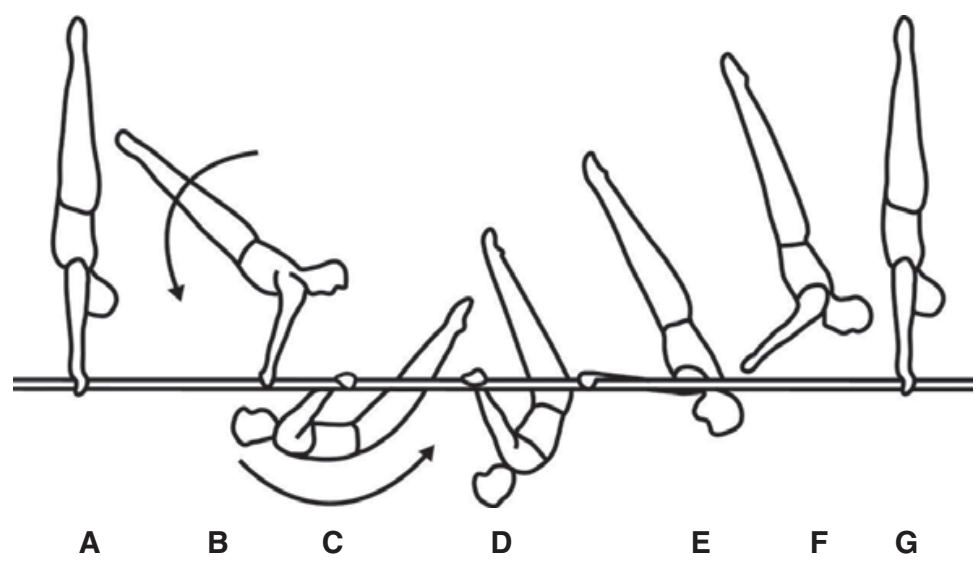

Figure 1 - The undersomersault from handstand to handstand on parallel bars (adapted from the FIG Code of Points, 2006).

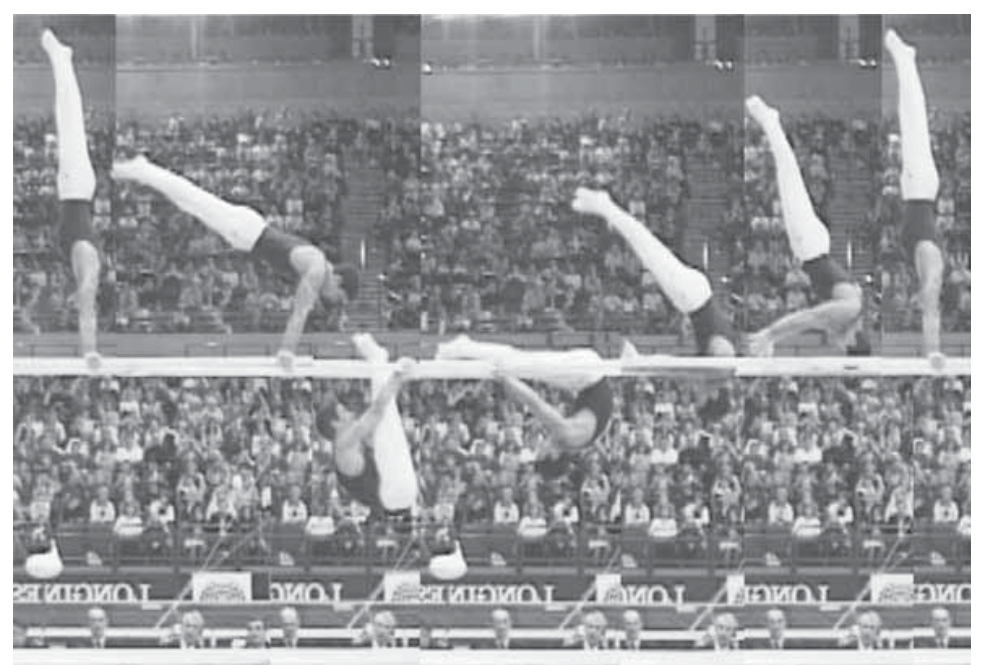

Figure 2 - The undersomersault from handstand to handstand performed with a "deep" hip flexion as the gymnast passes beneath the bars.

Previous research has investigated the undersomersault to handstand mount on parallel bars (Takei \& Dunn, 1996). The performances of 26 national gymnasts were divided into two groups by score and were then analyzed. It was found that the better performances were associated with a higher mass center position and greater vertical velocity at release. It was also found that in the poorer performances the gymnasts over-rotated before releasing the bars which lead to a poor body position on regrasp.

Limited work has been carried out on simulating swinging on parallel bars. A two-dimensional frontal plane model of the gymnast and bars has been used to look at the lateral movements of the bars during handstand (Linge, Hallingstad, \& Solberg, 2006). Although the model was able to simulate the lateral movements of the bars during these activities no data were presented for swinging in the sagittal plane. However, there are many examples in the literature of planar simulation models used to investigate swinging in the sagittal plane on high bar (Arampatzis \& Bruggemann, 1999; Hiley \& Yeadon, 2003a; Linge \& Hallingstad, 2002). To optimize technique using a simulation model, an appropriate cost function must be chosen. Depending on the nature of the cost function, different optimum techniques may be obtained. Typically in high bar swinging the amount of angular momentum at release for a dismount has been maximized (Hiley \& Yeadon, 2003a). However, in the study by Takei and Dunn (1996), excessive angular momentum appears to be undesirable for successful performance. The question arises, "What would happen to technique if maximizing angular momentum were the optimization criterion?" 
The aim of the current study was investigate the effect of cost function on optimum technique for the swing phase of the undersomersault. The techniques of two gymnasts are optimized with three different cost functions based on (a) minimizing peak joint torques at the hip and shoulder (b) producing a vertical path of the mass center leading up to release and (c) maximizing angular momentum about the mass center at release.

\section{Methods}

Subsections in Methods describe the protocol used to optimize the technique used in the swing phase of the undersomersault from handstand to handstand on parallel bars. Data collection was carried out using a three-dimensional motion capture system and the data from several undersomersault to handstand trials were processed to provide input to the computer simulation model and comparison data for the model evaluation. The model was initially used to obtain matching simulations of the undersomersault performances to determine model parameters. The matching simulations were then used as the starting point for optimizing the gymnast's technique (i.e., determine the joint angle time histories) using the different cost functions.

\section{Data Collection}

Data were collected on two senior male gymnasts competing at national level (Gymnast 1: mass $=61.2 \mathrm{~kg}$, height $1.65 \mathrm{~m}$; Gymnast 2: mass $=63.5 \mathrm{~kg}$, height $1.75 \mathrm{~m}$ ) to provide input data for the simulation model and provide comparison data with which to evaluate the model. Each gymnast gave informed consent (in accordance with the procedures of the university ethics committee) and each performed nine undersomersaults from handstand to handstand. All trials were recorded using 13 Vicon M2 cameras operating at $100 \mathrm{~Hz}$. Before data collection a volume centered on the parallel bars spanning $5 \mathrm{~m} \times$ $2 \mathrm{~m} \times 5 \mathrm{~m}$ was wand calibrated using the Vicon motion analysis system. Spherical reflective markers $25 \mathrm{~mm}$ in diameter were attached to the lateral side of the wrist, elbow, shoulder, hip, knee and ankle joint centers and toes on each side of the body. Offset measurements, using an anthropometer, were recorded from each marker center to the adjacent joint center for subsequent determination of joint centers. Markers were also attached to each side of the gymnast's head and on top of each parallel bar at its center. Three-dimensional marker coordinates were reconstructed and joint centers were determined, using the offset measurements, from which arm orientation and joint configuration angles were calculated (Yeadon, 1990a). The inertia parameters of each gymnast were calculated using the model of Yeadon (1990b) from a set of 95 anthropometric measurements taken on each gymnast.

All trials were also recorded with a standard $50 \mathrm{~Hz}$ digital video camcorder (Panasonic NV-GS200EB). Four international judges (FIG) scored each undersomersault from the video recordings. The highest and lowest scoring trials of each gymnast were chosen for further analysis. None of the chosen trials achieved the final handstand position without the use of strength.

\section{Simulation Model}

A three-segment planar model was used to simulate the swinging movement on the parallel bars (Hiley \& Yeadon, 2003a). The gymnast model included arm, torso, and leg segments which were given subject-specific inertia parameters. As flexing of the elbow or knee would lead to deductions from the judges, a single segment was sufficient to represent straight arms and another to represent straight legs. The elastic properties of the bars and gymnast were modeled as damped linear springs (Figure 3). The spring at the shoulder represented the stretch at the shoulder and any extension of the spine. Movement of the shoulder (gleno-humeral) joint center due to scapular rotation was represented by allowing the torso length to vary as a function of shoulder (arm elevation) angle (Begon, Wieber \& Yeadon, 2008). The model was driven using the joint angle time histories at the shoulder and hip (Figure 3, $\alpha$ and $\beta$, respectively) in the form of Fourier series.

\section{Matching Simulations}

To determine model parameters which could not be measured directly (e.g., spring coefficients and joint torques) and provide a starting point for the optimization

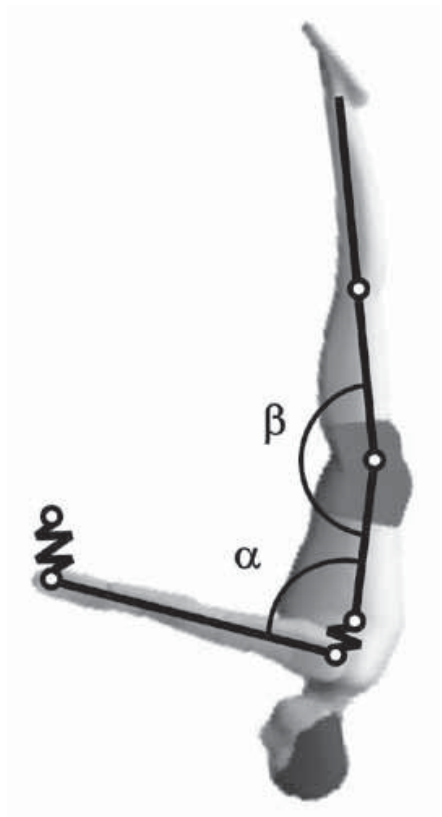

Figure 3 - Three segment simulation model of the gymnast and parallel bars with bar spring and shoulder spring. 
of technique (i.e., joint angle time histories), a close match between simulated and recorded performance was required for the two selected trials of each gymnast. The simulation model was implemented with the Simulated Annealing optimization algorithm (Goffe, Ferrier \& Rogers, 1994) to minimize the difference between the simulated and recorded performance. The matching cost function (equation 1) included terms for differences between simulated and recorded rotation angle, bar displacements and joint angle time histories at the hip and shoulder, normalized angular momentum at release and linear velocity at release (Table 1 ).

$$
\begin{gathered}
\mathrm{F}=10 \phi+200\left(\mathrm{x}_{\mathrm{b}}+\mathrm{z}_{\mathrm{b}}\right)+ \\
20\left(\mathrm{~h}+\dot{\mathrm{X}}_{\mathrm{cm}}+\dot{\mathrm{Z}}_{\mathrm{cm}}\right)+20(\alpha+\beta)
\end{gathered}
$$

where $\phi=$ root mean squared (RMS) difference in degrees between recorded and simulated rotation angle, $\mathrm{x}_{\mathrm{b}}$ and $\mathrm{z}_{\mathrm{b}}$ are the RMS differences between recorded and simulated bar displacements, $\mathrm{h}=$ absolute difference in normalized angular momentum at release between simulation and actual performance, $\dot{\mathrm{X}}_{\mathrm{cm}}$ and $\dot{\mathrm{Z}}_{\mathrm{cm}}$ are the absolute differences in linear velocity at release between simulation and actual performance, $\alpha$ and $\beta$ are the RMS differences between the joint angle time histories determined from the Fourier series and the actual performances. Angular momentum was normalized by dividing by $2 \pi$ times the moment of inertia of the body about its mass center when straight and multiplying by the time of flight to give the number of straight somersaults (SS) in flight.

Since the model was designed to simulate the swinging portion of the skill, each simulation was started once the angular velocity of the arm segment was in the positive direction (anticlockwise, Figure 2). The end of the simulation was defined as the point at which the gymnast lost contact with the bars. Initial conditions, including the initial angle, angular velocity and bar displacements, for each simulation were taken from the corresponding trial. During the matching process the following parameters were allowed to vary to obtain a match between the simulated and recorded performance. The horizontal and vertical bar stiffness and damping coefficients were allowed to vary to conform with the norms of the International Gymnastics Federation (FIG, 2006). The stiffness and damping coefficients of the shoulder spring were allowed to vary between 0 and $30,000 \mathrm{~N} \cdot \mathrm{m}^{-1}$ and between 0 and $5,000 \mathrm{~N} \cdot \mathrm{s} \cdot \mathrm{m}^{-1}$, respectively (Hiley and Yeadon, 2007). The masses of the arms and legs were allowed to vary independently by $\pm 5 \%$ and the torso mass was adjusted to maintain whole body mass, since density values were taken from the literature for the segmental mass calculations. The torso length parameter was allowed to vary between 0 and $0.15 \mathrm{~m}$. In addition variations in the initial conditions (rotation angle and angular velocity) were permitted (up to $6^{\circ}$ and $40^{\circ} \cdot \mathrm{s}^{-1}$ ) to compensate for any errors propagated during calculation. Finally, the coefficients which defined the Fourier series were allowed to vary to obtain a close match with the recorded joint angle time histories. All model parameters and initial conditions determined during the matching process remained fixed during the subsequent optimization of technique.

\section{Optimizations}

Three separate cost functions (minimization of peak joint torques, production of vertical mass center path and maximization of angular momentum at release) were used to optimize the gymnasts' technique during the swing phase of the undersomersault. All simulations started from the same position as in the matching process and finished once the torso segment had rotated $40^{\circ}$ past the vertical, since release should occur before this point in a good performance (Takei \& Dunn, 1996). The matching simulations were used to provide the initial estimates of the joint angle time histories. To produce realistic solutions the peak joint torques were limited to those determined from the matching simulations (Table 2). A reduced joint torque limit based on the results of the matching simulations (Table 2) was also used once the

Table 1 Mass center height and velocity for the four undersomersault to handstand performances

\begin{tabular}{lccccc}
\hline & \multicolumn{2}{c}{ Gymnast 1 } & & \multicolumn{2}{c}{ Gymnast 2 } \\
\cline { 2 - 3 } \cline { 6 - 7 } Variable & Best & Worst & & Best & Worst \\
\hline $\mathrm{CM}_{\mathrm{h} \text { release }}(\mathrm{m})$ & 0.177 & 0.187 & & 0.176 & 0.145 \\
$\mathrm{CM}_{\mathrm{h} \text { regrasp }}(\mathrm{m})$ & 0.642 & 0.595 & & 0.614 & 0.656 \\
$\mathrm{CM}_{\mathrm{h} \text { handstand }}(\mathrm{m})$ & 0.902 & 0.902 & & 1.018 & 1.018 \\
Vert. velocity & 3.115 & 2.902 & & 3.171 & 3.169 \\
Horiz. veloase $\left(\mathrm{m} \cdot \mathrm{s}^{-1}\right)$ & 0.097 & 0.164 & & 0.035 & 0.319 \\
Angular momentum $(\mathrm{SS})$ & 0.000 & 0.007 & & -0.004 & -0.002 \\
\hline
\end{tabular}

Note. $\mathrm{CM}_{\mathrm{h}}$ is mass center height. Angular momentum is measured in straight somersaults (SS) during flight. 


\begin{tabular}{|c|c|c|c|c|}
\hline \multirow[b]{2}{*}{ Variable } & \multicolumn{2}{|c|}{ Gymnast 1} & \multicolumn{2}{|c|}{ Gymnast 2} \\
\hline & Best & Worst & Best & Worst \\
\hline Peak shoulder torque $(\mathrm{N} \cdot \mathrm{m})$ : $\max$ & 374 & 402 & 354 & 429 \\
\hline $\min$ & -427 & -377 & -169 & -212 \\
\hline Peak hip torque $(\mathrm{N} \cdot \mathrm{m})$ : $\max$ & 93 & 191 & 213 & 184 \\
\hline $\min$ & -209 & -398 & -469 & -460 \\
\hline Shoulder torque limit $(\mathrm{N} \cdot \mathrm{m})$ : $\max$ & \multicolumn{2}{|c|}{405} & \multicolumn{2}{|c|}{430} \\
\hline $\min$ & \multicolumn{2}{|c|}{-430} & \multicolumn{2}{|c|}{-215} \\
\hline Hip torque limit $(\mathrm{N} \cdot \mathrm{m}): \max$ & \multicolumn{2}{|c|}{200} & \multicolumn{2}{|c|}{215} \\
\hline $\min$ & \multicolumn{2}{|c|}{-400} & \multicolumn{2}{|c|}{-470} \\
\hline Shoulder torque limit release $(\mathrm{N} \cdot \mathrm{m})$ & \multicolumn{2}{|c|}{ \pm 175} & \multicolumn{2}{|c|}{ \pm 175} \\
\hline Hip torque limit release $(\mathrm{N} \cdot \mathrm{m})$ & \multicolumn{2}{|c|}{ \pm 200} & \multicolumn{2}{|c|}{ \pm 200} \\
\hline
\end{tabular}

Note. Positive torques correspond to shoulder extension and hip flexion.

model had rotated through $250^{\circ}$ to reflect the reduced grip on the bars. In all three optimizations only the coefficients that defined the joint angle time histories were allowed to vary, all other model parameters obtained during the matching process remained fixed. The upper and lower bounds for the Fourier series coefficients were allowed to vary by $\pm 25 \%$ from the initial values.

The first optimization minimized a cost function based on the peak joint torques at the hip and shoulder joints while seeking an improved undersomersault performance through the use of penalties. The optimum technique was required to produce sufficient vertical velocity at release to achieve a mass center height in flight of at least $90 \%$ of the final handstand position measured above the bars. The $90 \%$ of handstand position was chosen as gymnasts regrasp the bars before handstand is reached and this also represented a significant improvement from the gymnast performances (i.e., would allow the gymnasts to perform the skill with straight arms). The vertical velocity required to reach $90 \%$ of handstand was calculated from the height of the mass center at release using the equations of motion under constant acceleration. The simulation incurred penalties if the horizontal velocity and normalized angular momentum at release exceeded the range spanned by the video analysis of the 18 trials of the gymnasts and the values reported for high scoring performances by Takei \& Dunn (1996) $(0.3 \mathrm{~m} / \mathrm{s}>$ horizontal velocity $>-0.2 \mathrm{~m} / \mathrm{s}$ and $0.025 \mathrm{SS}>$ normalized angular momentum $>-0.025 \mathrm{SS}$ ). An additional penalty was imposed for excessive hip flexion at release from the bars as this was likely to result in poor body configurations on regrasping the bars (Takei \& Dunn, 1996). The cost function was calculated at each integration interval during the upward phase of the swing (Figure 1D-F) until the end of the simulation. The lowest value obtained for the cost function during this period was returned to the optimization algorithm and defined the instant of release.

The second optimization effectively minimized horizontal velocity so as to produce a vertical path of the mass center during the upward swing to release. A root mean squared (RMS) difference was calculated between the angle of the resultant mass center velocity and the upward vertical from a rotation angle of $225^{\circ}$ until release. In addition to minimizing the RMS difference, exactly the same penalties used to produce an improved undersomersault performance in the first cost function were included (i.e., sufficient vertical velocity to achieve $90 \%$ of the final handstand position along with the constraints of horizontal velocity, angular momentum and hip angle at release).

The third optimization maximized the forward normalized angular momentum of the model at release from the bars. Since this skill was unlikely to regrasp the bars in handstand the time of flight was defined from release until the mass center was level with the bars. Similarly there was no penalty associated with horizontal velocity at release. A simulation model of aerial movement (Yeadon, Atha \& Hales, 1990) was used to determine what skills would be possible based on the increase in normalized angular momentum.

\section{Results}

The mass center release height and velocity were similar for the four performances (Table 1). In each of the four matching simulations the model was able to reproduce the whole body rotation angle to within $2^{\circ}$ (RMS difference), the bar displacements to within $0.005 \mathrm{~m}$ (RMS difference), the joint angle time histories to within $4^{\circ}$ (RMS difference), and the mass center velocity at release 
to within $1 \%$. The mean vertical stiffness and damping coefficients of the bar obtained in the four matching trials were $63,573 \mathrm{~N} \cdot \mathrm{m}^{-1}$ and $686 \mathrm{~N} \cdot \mathrm{s} \cdot \mathrm{m}^{-1}$, respectively. The mean horizontal stiffness and damping coefficients of the bar were considerably larger, due to the construction of the apparatus $\left(152,189 \mathrm{~N} \cdot \mathrm{m}^{-1}\right.$ and $\left.7,880 \mathrm{~N} \cdot \mathrm{s} \cdot \mathrm{m}^{-1}\right)$. The mean shoulder stiffness and damping coefficients were $14,308 \mathrm{~N} \cdot \mathrm{m}^{-1}$ and $1,656 \mathrm{~N} \cdot \mathrm{s} \cdot \mathrm{m}^{-1}$, respectively. The peak torques at the hip and shoulder joints during the matching simulations were generally similar for the two gymnasts with the exception that Gymnast 2 had lower peak shoulder flexion torques (Table 2).

In the first set of optimizations where the peak joint torques were minimized, the model was able to achieve sufficient vertical velocity at release while satisfying the criteria for a successful performance. This represents an improvement in performance of $25 \%$ and $36 \%$ in terms of the height achieved by the mass center for each gymnast, respectively. As expected the peak joint torques were lower than in the actual performances (Tables 3, 4; Figure 4-positive torques correspond to shoulder extension and hip flexion). The technique in the first set of optimized simulations (Figure 5b) differed from the technique of the gymnasts (Figure 5a) and more closely resembled the clear circle technique (Figure 1).

The second set of optimizations, in which the path of the mass center was encouraged to be vertical, was still able to achieve sufficient vertical velocity at release while satisfying the criteria for a successful performance (i.e., same level of improvement in performance as the first set of optimizations). Although the peak joint torques did not exceed the limits set from

Table 3 Gymnast 1: Release parameters and peak joint torques for the three sets of optimisations

\begin{tabular}{|c|c|c|c|c|c|c|}
\hline \multirow[b]{2}{*}{ Variable } & \multicolumn{2}{|c|}{ Optimization 1} & \multicolumn{2}{|c|}{ Optimization 2} & \multicolumn{2}{|c|}{ Optimization 3} \\
\hline & Best & Worst & Best & Worst & Best & Worst \\
\hline $\mathrm{CM}_{\mathrm{h} \text { release }}(\mathrm{m})$ & 0.196 & 0.201 & 0.230 & 0.223 & 0.245 & -0.046 \\
\hline Vert. velocity release $\left(\mathrm{m} \cdot \mathrm{s}^{-1}\right)$ & 3.57 & 3.54 & 3.38 & 3.40 & 4.34 & 4.46 \\
\hline Horiz. velocity release $\left(\mathrm{m} \cdot \mathrm{s}^{-1}\right)$ & -0.14 & 0.11 & -0.20 & -0.19 & 1.41 & 3.25 \\
\hline Angular momentum (SS) & -0.025 & -0.023 & -0.024 & -0.022 & -0.628 & -0.611 \\
\hline Max shoulder torque $(\mathrm{N} \cdot \mathrm{m})$ & 136 & 139 & 406 & 371 & 298 & 307 \\
\hline Min shoulder torque $(\mathrm{N} \cdot \mathrm{m})$ & -124 & -179 & -430 & -430 & -430 & -420 \\
\hline Max hip torque $(\mathrm{N} \cdot \mathrm{m})$ & 143 & 139 & 101 & 114 & 200 & 139 \\
\hline Min hip torque $(\mathrm{N} \cdot \mathrm{m})$ & -206 & -195 & -399 & -378 & -400 & -401 \\
\hline RMS vertical velocity $\left(^{\circ}\right)$ & 21 & 21 & 6 & 10 & 27 & 42 \\
\hline
\end{tabular}

Note. RMS vertical velocity is the RMS difference in degrees between the direction of the mass center velocity and the upward vertical leading up to release.

Table 4 Gymnast 2: Release parameters and peak joint torques for the three sets of optimisations

\begin{tabular}{|c|c|c|c|c|c|c|}
\hline \multirow[b]{2}{*}{ Variable } & \multicolumn{2}{|c|}{ Optimization 1} & \multicolumn{2}{|c|}{ Optimization 2} & \multicolumn{2}{|c|}{ Optimization 3} \\
\hline & Best & Worst & Best & Worst & Best & Worst \\
\hline $\mathrm{CM}_{\mathrm{h} \text { release }}(\mathrm{m})$ & 0.249 & 0.274 & 0.284 & 0.381 & 0.306 & 0.220 \\
\hline Vert. velocity release $\left(\mathrm{m} \cdot \mathrm{s}^{-1}\right)$ & 3.62 & 3.56 & 3.52 & 3.24 & 3.64 & 3.08 \\
\hline Horiz. velocity release $\left(\mathrm{m} \cdot \mathrm{s}^{-1}\right)$ & -0.10 & -0.08 & -0.19 & -0.20 & 1.41 & 1.24 \\
\hline Angular momentum (SS) & -0.017 & -0.019 & -0.023 & -0.023 & -0.408 & -0.404 \\
\hline Max shoulder torque $(\mathrm{N} \cdot \mathrm{m})$ & 383 & 281 & 392 & 417 & 376 & 430 \\
\hline Min shoulder torque $(\mathrm{N} \cdot \mathrm{m})$ & -138 & -126 & -192 & -217 & -196 & -214 \\
\hline Max hip torque $(\mathrm{N} \cdot \mathrm{m})$ & 165 & 195 & 189 & 215 & 210 & 215 \\
\hline Min hip torque $(\mathrm{N} \cdot \mathrm{m})$ & -200 & -226 & -474 & -475 & -467 & -409 \\
\hline RMS vertical velocity $\left({ }^{\circ}\right)$ & 21 & 21 & 7 & 8 & 29 & 31 \\
\hline
\end{tabular}


(a)

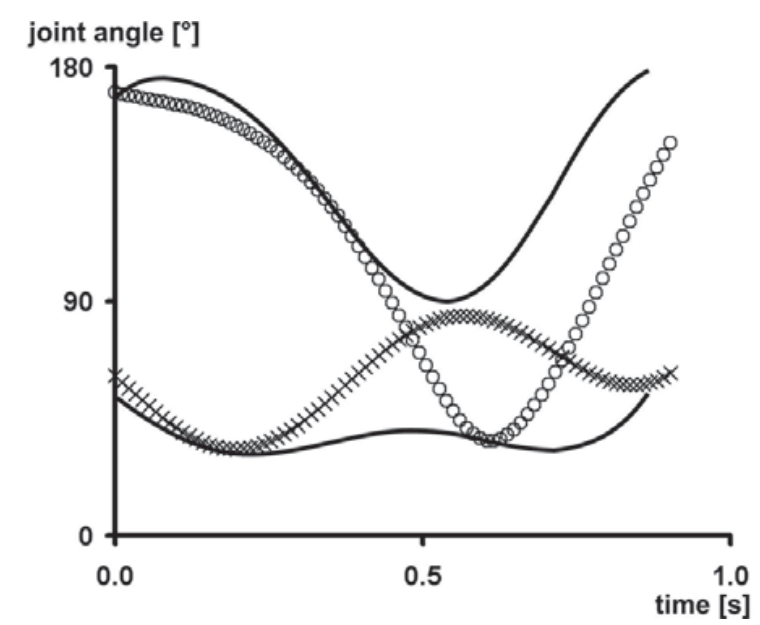

(b)

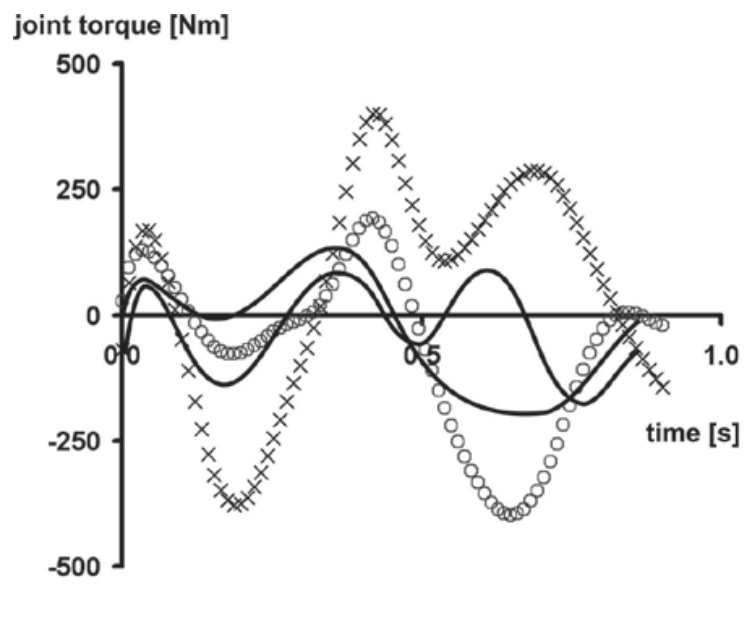

Figure 4 - Time histories of (a) joint angles and (b) joint torque at the hip (circles) and shoulder (crosses) for the matched and optimized (solid line) simulations of the worst trial of Gymnast 1 from the first set of optimizations.
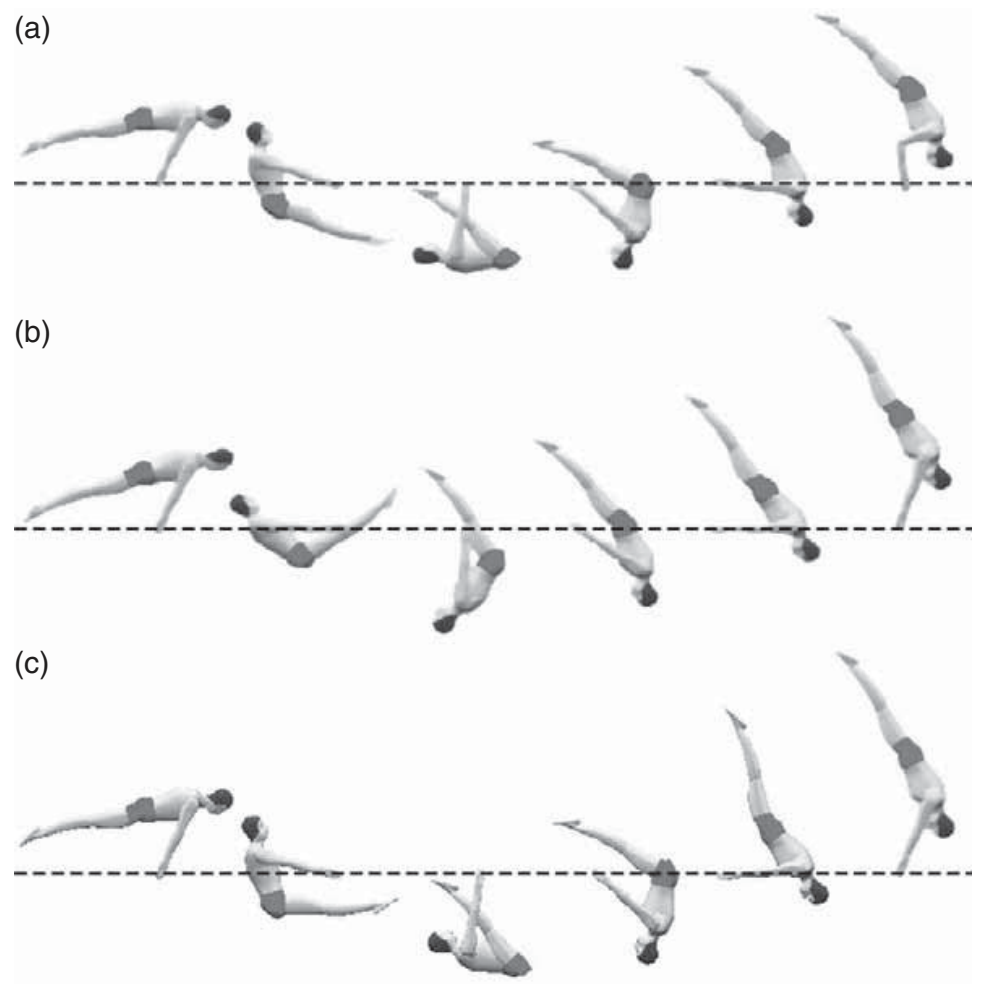

Figure 5 - Graphical sequences of (a) matched (regrasp position taken from video analysis), and optimum simulations (b) minimizing joint torques and (c) producing vertical mass center path of the best trial of Gymnast 2 .

the actual performances they were considerably higher than those obtained from the first set of optimizations (Figure 6; Tables 3, 4). The technique from the second set of optimizations (Figure 5c) closely resembled the technique of the two gymnast performances (Figure 5a). The main difference between the second set of optimum techniques and the gymnasts' technique was a more rapid hip extension over a slightly larger hip angle range in the optimized solutions (Figure 6a).

In the third set of optimizations, where the forward normalized angular momentum was maximized, considerably more angular momentum at release was 
produced (Tables 3,4) when compared with the gymnasts' performances. This was accompanied by a considerable increase in horizontal velocity, compared with the other two sets of optimizations, as this was no longer constrained by the cost function. For Gymnast 1 the model was able to produce more normalized angular momentum than Gymnast 2, and would have been sufficient to perform a "cast to straddle cut" (Figure 7a). The model produced sufficient angular momentum for Gymnast 2 to perform a similar skill, but in a tucked position and without the straddle cut (Figure 7b).

(a)

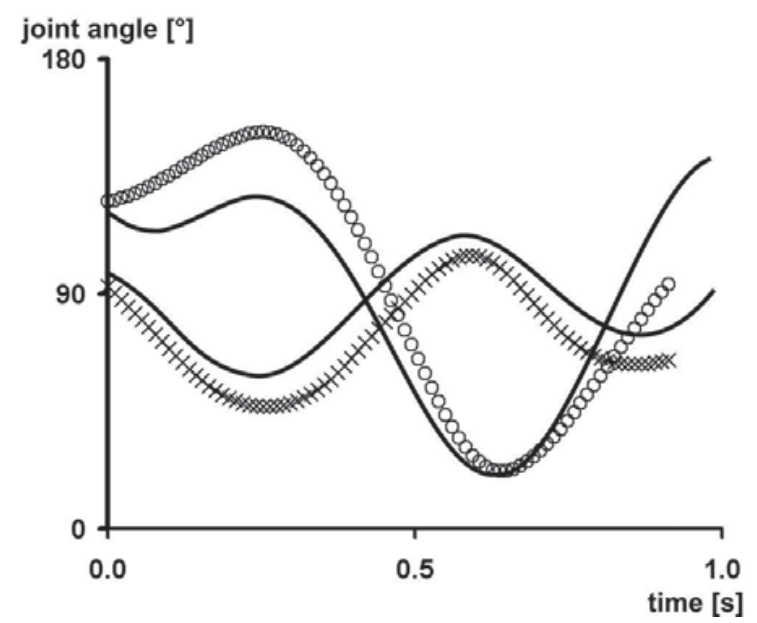

\section{Discussion}

The aim of the paper was to investigate the effect of the cost function on optimal technique for the undersomersault class of parallel bars skills. In the first two optimizations the goal was to improve upon the performance of the undersomersault to handstand of two elite gymnasts. In all cases the first set of optimizations was able to improve upon the gymnasts' performances with the mass center able to reach over $90 \%$ of handstand height in the flight phase, which would enable the gymnasts to regrasp the

(b)

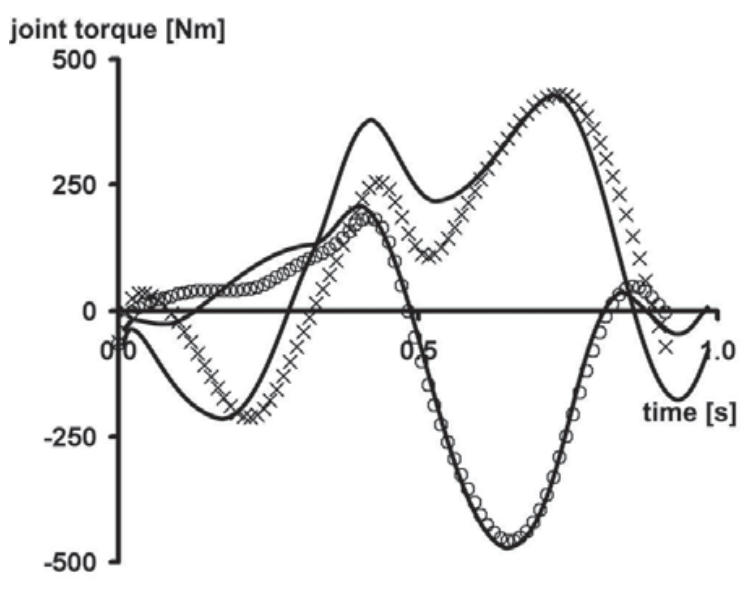

Figure 6 - Time histories of (a) joint angles and (b) joint torque at the hip (circles) and shoulder (crosses) for the matched and optimized (solid line) simulations of the worst trial of Gymnast 2 from the second set of optimizations.
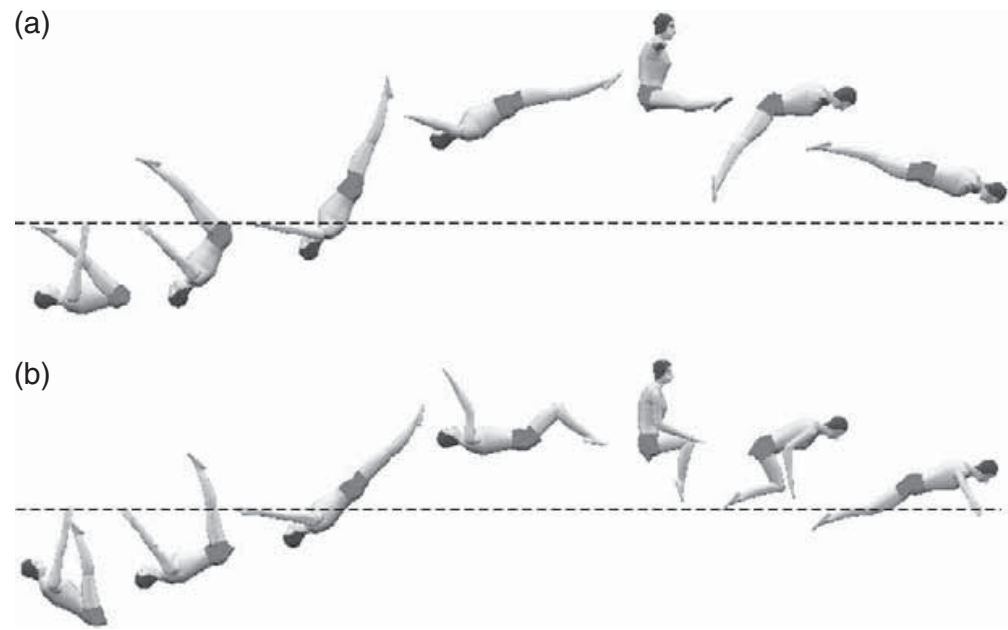

Figure 7 - Graphical sequences of the new skills that could be performed from the undersomersault optimized for angular momentum of (a) Gymnast 1 and (b) Gymnast 2. 
bars with straight arms (Figure 5). The average heights achieved by the two gymnasts in their actual performances were $72 \%$ and $66 \%$ of handstand, respectively. The improvement was achieved through a combination of increased vertical velocity and an increase in height of the mass center at release (Tables $1,3,4)$. In the case of the optimum technique which closely resembled the gymnasts' technique, this was achieved through a more rapid hip extension over a slightly larger range (Figure 6). Although similar demands in terms of a successful performance were required, the cost functions used in the first two optimizations were very different.

A limitation of the current study is the use of fixed joint torque limits. Although it would be desirable to have limits based on a joint torque - angle - angular velocity relationship, the results do not depend on excessively large joint torques compared with the actual performances (Figures 4 and 6). Inspection of the joint angular velocity time histories revealed that peak angular velocity occurred at periods of relatively low joint torque for the first and third set of optimizations. In the second set of optimizations, at the shoulder joint, there were instances of larger joint torques in comparison with the first set. It is expected that the inclusion of a torque-angle-angular velocity relationship might make small changes to the optimal technique, but would not affect the conclusions regarding the two techniques. It could also be argued that a further limitation of the study was constraining the optimization to the swing phase of the skill. However, since simulations started from the beginning of this almost stationary phase future studies could address this aspect of the technique by allowing the initial conditions (i.e., configuration and angular velocity) to vary as well as the joint angle time histories.

The minimization of joint torques may be an appropriate optimization criterion where the gymnast strength limits are approached during the movement. When minimizing the joint torques a technique similar to the backward clear circle technique was obtained. This finding supports the coaching recommendation that the clear circle technique is adopted in the initial stages of learning, as it is physically less demanding (Figure 4b).

In the case of the stoop stalder technique it is clear that minimizing peak joint torques is not the goal of the gymnast. The stoop stalder technique was obtained from the optimization that encouraged a vertical path of the mass center as the gymnast approached release (Figure 8). As outlined in the introduction, the advantages of such a linear mass center path are consistency of performance and the potential for increasing the complexity of the skill with additional turns during the flight phase. Gymnastics is a sport that relies on consistency and the continuing development of new and more complex skills. In effect the clear circle technique may be redundant, beyond the initial stages of learning, as it does not have the benefits for skill progression that the stoop stalder has.

The third set of optimizations also demonstrates that gymnast's technique is more often based on task requirements than minimization of torques or energy. Replacing the requirements for a good undersomersault to handstand with the maximization of normalized angular momentum resulted in sufficient changes in technique to (a)

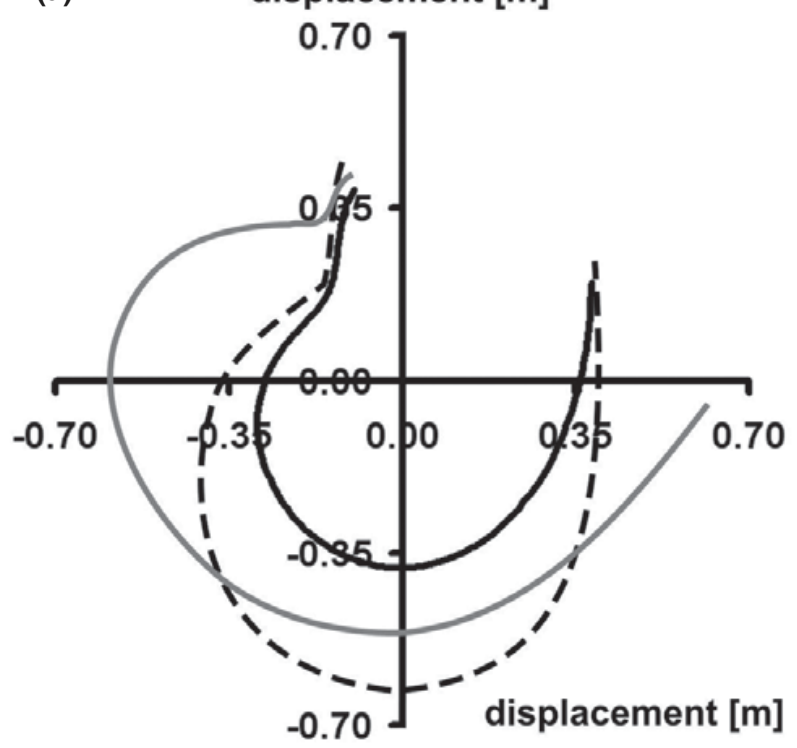

(b)

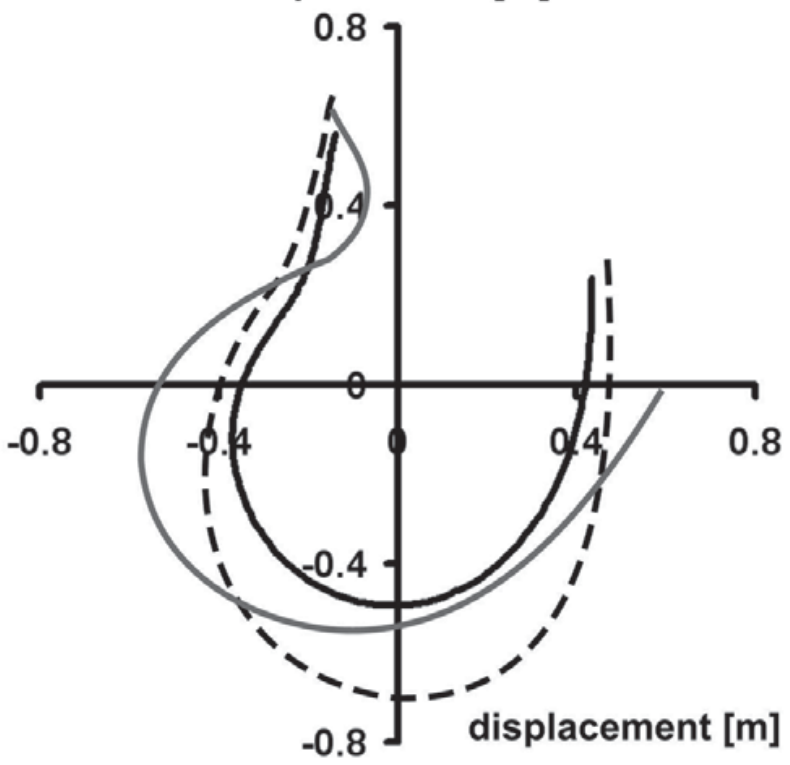

Figure 8 - Path of the mass center for optimization 1 (solid black), optimization 2 (dashed line) and optimization 3 (solid gray) for (a) the best trial of Gymnast 1 and (b) the best trial of Gymnast 2 . 
produce a completely new skill (although still within the same family of under swings). For Gymnast 1 sufficient angular momentum was produced to perform a "cast to straddle cut." This is classified as a D element in the FIG code of Points (2006) making it more difficult than the undersomersault to handstand, which is classified as a $\mathrm{C}$ (skills are ranked from A to E).

In this article, optimizations have been based upon minimizing peak joint torque, having a vertical release velocity, and maximizing normalized angular momentum in undersomersaulting movements. Each of these criteria produces a characteristic technique associated with the particular skill. The identification of an appropriate criterion for a given skill is not always a simple matter since the over-riding factor might be strength (optimization 1), skill development (optimization 2), mechanical (optimization 3) or something else such as timing (Hiley $\&$ Yeadon, 2003). Indeed it may not always be possible to explain technique in terms of a single optimization criterion since sometimes there may be a number of competing factors that are relevant and important.

\section{References}

Arampatzis, A., \& Brüggemann, G-P. (1999). Mechanical energetic processes during the giant swing exercise before dismounts and flight elements on the high bar and the uneven parallel bars. Journal of Biomechanics, 32, 811-820.

Begon, M., Wieber, P-B., \& Yeadon, M.R. (2008). Kinematics estimation of straddled movements on high bar from a limited number of skin markers using a chain model. Journal of Biomechanics, 41, 581-586.
Davis, J. (2005). Undersomersaults on parallel bars. Gym Craft, 14, 6-7.

Fédération Internationale de Gymnastique. (2006). Code of Points. Moutier, Switzerland: F.I.G.

Goffe, W.L., Ferrier, G.D., \& Rogers, J. (1994). Global optimization of statistical functions with simulated annealing. Journal of Econometrics, 60, 65-99.

Hiley, M.J., \& Yeadon, M.R. (2003). The margin for error when releasing the high bar for dismounts. Journal of Biomechanics, 36, 313-319.

Hiley, M.J., \& Yeadon, M.R. (2003a). Optimum technique for generating angular momentum in accelerated backward giant circles prior to a dismount. Journal of Applied Biomechanics, 19, 119-130.

Hiley, M.J., \& Yeadon, M.R. (2007). Optimization of backward giant circle technique on the asymmetric bars. Journal of Applied Biomechanics, 23, 300-308.

Linge, S., \& Hallingstad, O. (2002). Gymnastics - a new 2D model of the high bar including endpoint movement. Sports Engineering, 5, 81-92.

Linge, S., Hallingstad, O., \& Solberg, F. (2006). Modelling the parallel bars in Men's Artistic Gymnastics. Human Movement Science, 25, 221-237.

Takei, Y., \& Dunn, J.H.(1996). A comparison of techniques used by elite gymnasts in performing the basket-to-handstand mount. Journal of Sports Sciences, 14, 269-279.

Yeadon, M.R. (1990a). The simulation of aerial movement - I. The determination of orientation angles from film data. Journal of Biomechanics, 23, 59-66.

Yeadon, M.R. (1990b). The simulation of aerial movement - II. A mathematical inertia model of the human body. Journal of Biomechanics, 23, 67-74.

Yeadon, M.R., Atha, J., \& Hales, F.D. (1990). The simulation of aerial movement-IV: A computer simulation model. Journal of Biomechanics, 23, 85-89. 